\title{
Bragg Diffraction of Microcavity Polaritons by a Surface Acoustic Wave
}

\author{
Kikuo Cho, ${ }^{1, *}$ Kazunori Okumoto, ${ }^{1}$ N. I. Nikolaev, ${ }^{2}$ and A. L. Ivanov ${ }^{2}$ \\ ${ }^{1}$ Graduate School of Engineering Science, Osaka University, 1-3 Machikaneyama, Toyonaka 560-8531, Japan \\ ${ }^{2}$ Department of Physics and Astronomy, Cardiff University, Cardiff CF24 3YB, United Kingdom
}

(Received 22 December 2004; published 10 June 2005; corrected 13 June 2005)

\begin{abstract}
Bragg scattering of polaritons by a coherent acoustic wave is mediated and strongly enhanced by the exciton states resonant with the acoustic and optic fields in the intraband and interband transitions, respectively. In this case, in contrast with conventional acousto-optics, the resonantly enhanced Bragg spectra reveal the multiple orders of diffracted light. For polaritons in GaAs microcavities driven by a surface acoustic wave of $\nu_{\mathrm{SAW}}=1 \mathrm{GHz}$ and $I_{\mathrm{ac}} \lesssim 100 \mathrm{~W} / \mathrm{cm}^{2}$ the main acoustically induced band gap can be as large as $\Delta_{\mathrm{ac}}^{\mathrm{MC}} \simeq 0.6 \mathrm{meV}$ and the Bragg replicas up to $n=3$ can be observed.
\end{abstract}

DOI: 10.1103/PhysRevLett.94.226406

Back in 1933, Léon Brillouin, a founder of acoustooptics, described for the first time nonperturbative solutions of the Maxwell wave equation for the dielectric constant $\varepsilon$ harmonically modulated by an acoustic wave $\left\{\mathbf{k}, \Omega_{\mathbf{k}}^{\text {acc }}\right\}$, i.e., for $\varepsilon=\varepsilon_{b}+\delta \varepsilon_{b} \cos \left(\Omega_{\mathbf{k}}^{\mathrm{ac}} t-\mathbf{k r}\right) \quad$ [1]. Formally, the eigenvalues and eigenstates of the above equation are given in terms of the Mathieu functions and yield an infinite sequence of the alternating allowed and forbidden energy bands in momentum space. While the energy structure is akin to the electron energy bands, which appear in the presence of a periodic (atomic) potential, a very particular feature of the harmonic potential is that the forbidden energy bands, i.e., the acoustically induced energy gaps $\Delta_{\mathrm{ac}}^{(n)}(n=1,2, \ldots)$ in the photon spectrum, extremely effectively close up with the increasing energy number $n: \Delta_{\mathrm{ac}}^{(n)} \propto\left(\delta \varepsilon_{b}\right)^{n}$. This is an inherent property of the Mathieu wave functions [2]. The acousto-optic science deals with $\delta \varepsilon_{b}=4 \pi \chi_{\gamma-\mathrm{ac}}^{(2)} I_{\mathrm{ac}}^{1 / 2}$, where $I_{\mathrm{ac}}$ is the intensity of the acoustic wave and $\chi_{\gamma-\mathrm{ac}}^{(2)}$ is a second-order nonresonant acousto-optic susceptibility $[3,4]$. The nonresonant acousto-optic nonlinearities are small, so that usually $\delta \varepsilon_{b} \sim 10^{-4}-10^{-3}$ (for GaAs, e.g., $\delta \varepsilon_{b} \simeq 1.6 \times 10^{-3}$ for $I_{\mathrm{ac}}=100 \mathrm{~W} / \mathrm{cm}^{2}$ ). As a result, the conventional acousto-optics, both fundamental and applied, attributes the Bragg diffraction of the light field by a sound wave to the one-phonon transition only, i.e., to the main acoustically induced gap $n=1$ [3-6].

The nonresonant acousto-optic nonlinearities, which give rise to the acoustically induced diffraction grating, is due to the photoelastic effect. In sharp contrast with this mechanism, the resonant acousto-optic effect $[7,8]$ for bulk and microcavity (MC) polaritons deals with a quantum diffraction of optically induced, virtual excitons by the coherent acoustic wave. In this case the interaction of two "matter" waves, the excitonic polarization and the acoustic field, is much more effective than the nonresonant photoelastic coupling of photons with acoustic phonons. At the same time the photon component of optically dressed excitons, i.e., of polaritons, can be large enough to ensure an efficient resonant conversion "photon $\leftrightarrow$ exciton". This
PACS numbers: 71.36.+c, 42.65.Es, 43.60.+d

explains the origin of the resonant, exciton-mediated acousto-optic nonlinearity, $\delta \varepsilon_{\gamma-\mathrm{ac}}^{\mathrm{x}} \gg \delta \varepsilon_{b}$. For GaAsbased structures at low temperatures, $\delta \varepsilon_{\gamma-\mathrm{ac}}^{\mathrm{x}}$ can be as large as $\delta \varepsilon_{\gamma-\mathrm{ac}}^{\mathrm{x}} \sim 0.1$. Thus the resonant acousto-optics of polaritons deals with the microscopic mechanism fundamentally different from the photoelastic effect.

In this Letter we propose and calculate the acousto-optic Bragg scattering of surface acoustic wave (SAW)-driven polaritons. The MC polariton eigenstates in GaAs $\lambda$ cavities [9-11] are relevant to the proposed Bragg spectroscopy, due to compatibility of GaAs microcavities with the SAW technique [12] and due to a possibility to realize onedimensional geometry for the resonant, SAW-mediated interaction of two counter-propagating MC polaritons by using $\nu_{\mathrm{SAW}} \lesssim 3 \mathrm{GHz}$ [8]. As we demonstrate below, the Bragg spectra of $\mathrm{MC}$ polaritons are resonantly enhanced by the quantum well $(\mathrm{QW})$ exciton state and consist of the well-developed multiple replicas with a robust structure.

For the acousto-optic spectroscopy of polaritons, both resonant interactions, the exciton-photon coupling and the interaction of excitons with the acoustic pump wave, should be treated nonperturbatively (strong coupling regime) and on an equal basis. Being applied to the Bragg scattering of MC polaritons, the acousto-optic polariton macroscopic equations [7] are given by

$$
\begin{gathered}
{\left[\frac{\partial^{2}}{\partial x^{2}}+\frac{\partial^{2}}{\partial z^{2}}-\frac{\varepsilon_{b}(z)}{c^{2}} \frac{\partial^{2}}{\partial t^{2}}\right] E=\frac{4 \pi}{c^{2}} \frac{\partial^{2}}{\partial t^{2}} P+J_{\mathrm{ext}}} \\
{\left[\frac{\partial^{2}}{\partial t^{2}}+2 \gamma_{\mathrm{x}} \frac{\partial}{\partial t}+\omega_{\mathrm{T}}^{2}-\frac{\hbar \omega_{\mathrm{T}}}{M_{\mathrm{x}}} \frac{\partial^{2}}{\partial x^{2}}\right.} \\
\left.-4 m_{k}^{\mathrm{x}} \omega_{\mathrm{T}} \cos \left(\Omega_{k}^{\mathrm{ac}} t-k x\right)\right] P \\
=\Omega_{\mathrm{x}-\gamma}^{2} E
\end{gathered}
$$

where $E$ and $P$ are the light field and excitonic polarization, respectively, $J_{\text {ext }}$ is a source of the external optical wave necessary for the Bragg scattering problem, $\hbar \omega_{\mathrm{T}}$ is the energy position of the exciton line, $M_{\mathrm{x}}$ is the in-plane translational mass of QW excitons, $\Omega_{\mathrm{x}-\gamma}$ is the matrix element of the QW-exciton-MC-photon interaction, and $2 \gamma_{\mathrm{x}}=1 / T_{1}=2 / T_{2}$ is the rate of incoherent scattering of 
QW excitons. We assume a Cartesian coordinate system with the in-plane $x$ axis along the SAW wave vector $\mathbf{k}$, the $z$ axis along the MC growth direction, and the exciton and light fields linearly polarized along the in-plane $y$ axis. In this case the SAW, which has both transverse and longitudinal displacement components, $u_{x}$ and $u_{z}$, is elliptically polarized in the $x-z$ plane (the sagittal plane).

The SAW-induced modulation of the excitonic polarization is characterized by the coupling coefficient $m_{k}^{\mathrm{x}}=$ $I_{\mathrm{ac}}^{1 / 2}\left[\left|u_{x}\right| /\left(\left|u_{x}\right|^{2}+\left|u_{z}\right|^{2}\right)^{1 / 2}\right]\left|m_{\mathrm{x}-\mathrm{ac}}^{\mathrm{DP}}+i \gamma_{\mathrm{saw}} m_{\mathrm{x}-\mathrm{ac}}^{\mathrm{PE}}\right|$, where $m_{\mathrm{x}-\mathrm{ac}}^{\mathrm{DP}}$ and $m_{\mathrm{x}-\mathrm{ac}}^{\mathrm{PE}}$ are the deformation potential (DP) and piezoelectric (PE) matrix elements of exciton-SAW interaction, respectively, and $\gamma_{\text {saw }}=\left|u_{z}\right| /\left|u_{x}\right| \exp \left(i \delta_{\text {saw }}\right)$. The phase shift $\delta_{\text {saw }}$ between $u_{x}$ and $u_{z}$ is nearly $\pi / 2$ [12], so that both interaction channels interfere constructively. However, because $m_{\mathrm{x}-\mathrm{ac}}^{\mathrm{DP}} \gg m_{\mathrm{x}-\mathrm{ac}}^{\mathrm{PE}}\left(\right.$ for $\left.\nu_{\mathrm{SAW}} \lesssim 10 \mathrm{GHz}\right)$ and $\left|u_{z}\right| /\left|u_{x}\right| \simeq 1.4$ [13], the DP mechanism with $m_{\mathrm{x}-\mathrm{ac}}^{\mathrm{DP}}=$ $D_{\mathrm{x}} /\left(2 \hbar^{2} \rho v_{\mathrm{s}}^{3}\right)^{1 / 2}$ is strongly dominant over the PE one. Here, $\rho$ is the crystal density, $v_{\mathrm{s}}$ is the SAW velocity, and $D_{\mathrm{x}}$ is the exciton-LA-phonon DP. The above hierarchy of the interaction mechanisms is due to the charge neutrality of excitons: In contrast, the electron-SAW interaction is dominated by the PE coupling.

The $z$-dependent background dielectric function $\varepsilon_{b}(z)$ on the left-hand side (1.h.s.) of Eq. (1) refers to a stack of layers which form the MC structure we analyze, i.e., a GaAs $\lambda$ cavity with an embedded InGaAs QW, symmetrically sandwiched in between two identical AlGaAs/AlAs Bragg reflectors. The Bragg reflectors give rise to the transverse optical confinement and, at the same time, ensure an optical coupling of the external light wave with the in-plane polariton quasieigenstates. At first, however, in order to visualize the quasienergy spectrum of SAW-driven MC polaritons, we assume $100 \%$ optical confinement (i.e., replace the Bragg reflectors by perfect mirrors), no external light source [i.e., $J_{\mathrm{ext}}=0$ on the right-hand side (r.h.s.) of Eq. (1)], and no damping of the exciton states [i.e., $\gamma_{\mathrm{x}}=0$ on the 1.h.s. of Eq. (2)]. In this case the polariton spectrum is characterized by the MC Rabi frequency $\Omega_{\mathrm{x}}^{\mathrm{MC}}=$ $2\left(\pi / \varepsilon_{b}\right)^{1 / 2} \Omega_{\mathrm{x}-\gamma}$. In Fig. 1 we plot the quasienergy spectrum of lower-branch (LB) polaritons in a zero detuning MC with $\hbar \Omega_{\mathrm{x}}^{\mathrm{MC}}=3.7 \mathrm{meV}$, driven by the SAW of $\nu_{\mathrm{SAW}}=1 \mathrm{GH} z$ and $I_{\mathrm{ac}}=10 \mathrm{~W} / \mathrm{cm}^{2}$.

The quasienergy spectrum, which is calculated by the method developed in Ref. [7] for bulk polaritons and cannot be interpreted in terms of the Mathieu functions, shows the acoustically induced band gaps $\Delta_{\mathrm{ac}}^{\mathrm{MC}(\mathrm{n})} \propto I_{\mathrm{ac}}^{n / 2}$, due to the $n=1,2, \ldots$ phonon-assisted transitions. Because the energy of SAW phonons is very small, $h \nu_{\mathrm{SAW}} \simeq$ 4.1 $\mu \mathrm{eV}$ only, the acoustically induced spectral gaps refer to the in-plane counter-propagating MC polariton states $\left\{n k / 2, \omega_{\mathrm{LB}}^{\mathrm{MC}}(n k / 2)\right\}$ and $\left\{-n k / 2, \omega_{\mathrm{LB}}^{\mathrm{MC}}(n k / 2)\right\}$ (see the nearly horizontal arrows in Fig. 1), where $\omega_{\mathrm{LB}}^{\mathrm{MC}}\left(p_{\|}\right)$with $p_{\|}= \pm n k / 2$ is the LB solution of the dispersion equation $c^{2} p_{\|}^{2} / \varepsilon_{b}+\omega_{\mathrm{T}}^{2}-\omega^{2}=\left(\omega \Omega_{\mathrm{x}}^{\mathrm{MC}}\right)^{2} /\left(\omega_{\mathrm{T}}^{2}+\hbar \omega_{\mathrm{T}} p_{\|}^{2} / M_{\mathrm{x}}-\omega^{2}\right)$.

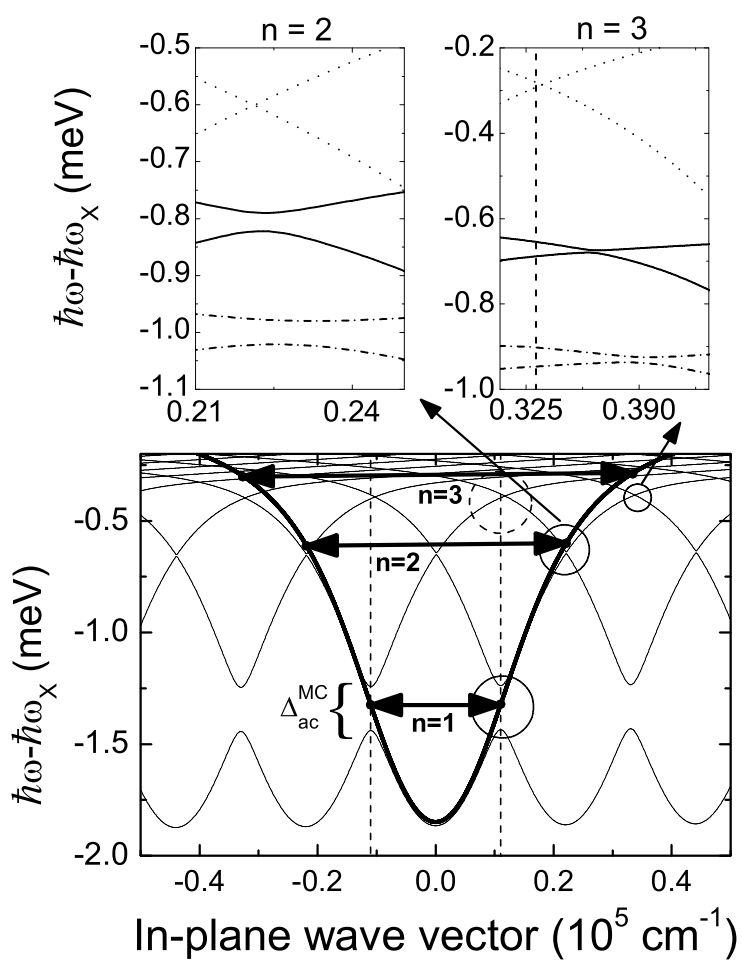

FIG. 1. The acoustically induced quasienergy spectrum of polaritons in a GaAs $\lambda$ microcavity driven by the SAW of $\nu_{\mathrm{SAW}}=1 \mathrm{GHz}, k=2.2 \times 10^{4} \mathrm{~cm}^{-1}, I_{\mathrm{ac}}=10 \mathrm{~W} / \mathrm{cm}^{2}, v_{\mathrm{s}}=$ $2.9 \times 10^{5} \mathrm{~cm} / \mathrm{s}$, and $D_{\mathrm{x}}=12 \mathrm{eV}$ (thin solid lines). The initial, acoustically unperturbed lower dispersion branch of MC polaritons is shown by the bold solid line. The MC Rabi energy is $\hbar \Omega_{\mathrm{x}}^{\mathrm{MC}}=3.7 \mathrm{meV}$. The magnified energy bands relevant to the resonant $n=2$ and 3 transitions are plotted in the 1.h.s. and r.h.s. upper row enlarged areas, respectively, for $I_{\mathrm{ac}}=0$ (dotted lines), $I_{\mathrm{ac}}=50 \mathrm{~W} / \mathrm{cm}^{2}$ (solid lines), and $I_{\mathrm{ac}}=100 \mathrm{~W} / \mathrm{cm}^{2}$ (dashdotted lines). The vertical dashed lines show the boundaries of the acoustically induced first Brillouin zone.

The main acoustically induced band gap in the LB spectrum is given by $\Delta_{\mathrm{ac}}^{\mathrm{MC}} \equiv \Delta_{\mathrm{ac}}^{\mathrm{MC}(\mathrm{n}=1)}=2\left|m_{k}^{\mathrm{x}}\right| \varphi^{\mathrm{MC}}(k / 2)$, where $\varphi^{\mathrm{MC}}(k / 2)=\left(\Omega_{\mathrm{x}}^{\mathrm{MC}}\right)^{2} /\left\{\left(\Omega_{\mathrm{x}}^{\mathrm{MC}}\right)^{2}+4\left[\omega_{\mathrm{T}}-\omega_{\mathrm{LB}}^{\mathrm{MC}}(k / 2)\right]^{2}\right\} \quad$ is the excitonic component of the polariton states $p_{\|}=$ $\pm k / 2$ resonantly coupled via one-SAW-phonon transition. Even for modest SAW intensities the main gap is large, so that $\Delta_{\mathrm{ac}}^{\mathrm{MC}} \gg \nu_{\mathrm{SAW}}$. For example, $I_{\mathrm{ac}}=100 \mathrm{~W} / \mathrm{cm}^{2}$ yields $\Delta_{\mathrm{ac}}^{\mathrm{MC}} \simeq 130 \mathrm{GHz}$. Note, that the conventional acoustooptics deals with the acoustically induced stop gap $\Delta_{\gamma-\text { ac }}$ less than the SAW frequency (usually, $\Delta_{\gamma-\mathrm{ac}} \lesssim 100 \mathrm{MHz}$ ) $[4,12]$. Furthermore, the acoustic band gaps associated with the two-phonon and three-phonon transitions in the MC polariton spectrum are also well developed, so that $\Delta_{\mathrm{ac}}^{\mathrm{MC}(\mathrm{n}=1)}: \Delta_{\mathrm{ac}}^{\mathrm{MC}(\mathrm{n}=2)}: \Delta_{\mathrm{ac}}^{\mathrm{MC}(\mathrm{n}=3)}=1: 0.08: 0.03$ for $I_{\mathrm{ac}}=$ $100 \mathrm{~W} / \mathrm{cm}^{2}$ (see the enlarged areas on the top row of Fig. 1). This is in a sharp contrast with the traditional acousto-optic schemes of the Bragg scattering. All the above features of the quasienergy spectrum of SAW-driven MC polaritons are due to the resonant, exciton-mediated acousto-optic susceptibility. 
Because of the periodicity of the acoustic wave, the quasienergy spectrum can also be interpreted in terms of an extended Brillouin zone, with the band boundaries at $p_{\|} \simeq \pm n k / 2$. The first acoustically induced Brillouin zone is shown in Fig. 1 by the vertical dashed lines. The energy band boundaries, where the spectral gaps arise and develop with increasing $I_{\mathrm{ac}}$, can be probed in Bragg scattering, by changing the incidence angle $\alpha$ of the external optical wave. At this point we return to Eqs. (1) and (2) applied to a realistic MC structure with the Bragg reflectors consisting of 34 alternating $\mathrm{AlAs}$ and $\mathrm{Al}_{0.13} \mathrm{Ga}_{0.87} \mathrm{As} \lambda / 4$ layers. The MC polariton quasieigenstates can now be excited by the external light field, $E_{\text {inc }}=E_{\text {inc }}^{(0)} e^{-i \omega t}$ [due to the $J_{\text {ext }}$ term on the r.h.s. of Eq. (1)], and can decay or scatter into the external electromagnetic modes.

In order to calculate the SAW-induced Bragg diffraction of optically excited MC polaritons, we use the Green function technique developed in Ref. [14]. Because the in-plane wave vector $p_{\|}$is conserved in the propagation of the light field through an optically transparent planar structure, one defines the MC-photon Green function $g\left(z, z^{\prime} ; \omega\right)$ by the equation

$$
\frac{d^{2}}{d z^{2}} g\left(z, z^{\prime} ; \omega\right)+\kappa^{2} g\left(z, z^{\prime} ; \omega\right)=-4 \pi \delta\left(z-z^{\prime}\right),
$$

where $\kappa^{2}=(\omega / c)^{2} \varepsilon_{b}(z)-p_{\|}^{2}$. The function $g\left(z, z^{\prime} ; \omega\right)$, which satisfies the Maxwellian boundary conditions, is evaluated numerically with the use of $\varepsilon_{b}(z)$ relevant to the MC structure we analyze. As a next step, we substitute in Eqs. (1) and (2) a Fourier expansion of the $E$ and $P$ fields in terms of the in-plane quasiwave vector $p_{\|}+\ell k$ and quasifrequency $\omega+2 \pi \ell \nu_{\mathrm{SAW}}$, and evaluate the SAWmediated acousto-optic susceptibility matrix $\chi_{\ell, \ell^{\prime}}: P_{\ell}=$ $\sum \ell^{\prime} \chi_{\ell, \ell^{\prime}} E_{\ell^{\prime}}(\ell=0, \pm 1, \ldots$, i.e., $\ell= \pm n$ corresponds to the $n$-phonon transition). The acoustically induced polarization harmonics $P_{\ell}$ give rise to the Bragg signal:

$$
E_{\ell}=E_{\ell}^{(0)}+q_{\ell}^{2} \int \mathrm{d} z^{\prime} g\left(z, z^{\prime}, \omega_{\ell}\right) \sum_{\ell^{\prime}} \chi_{\ell, \ell^{\prime}} E_{\ell^{\prime}}\left(z^{\prime}\right)
$$

where $q_{\ell}^{2}=\left(\omega+2 \pi \ell \nu_{\mathrm{SAW}}\right)^{2} / c^{2}, E_{\ell}=E_{\ell}(z)$ is the signal light field, and $E_{\ell}^{(0)}=E_{\ell}^{(0)}(z)$ is the incoming light field induced by $E_{\text {inc }}$. The field $E_{\ell}^{(0)}(z)$ is calculated by using the photon Green function defined by Eq. (3). The integration on the r.h.s. of Eq. (4) is over the QW thickness, so that one can approximate $E_{\ell^{\prime}}\left(z^{\prime}\right)$ by $E_{\ell^{\prime}}\left(z^{\prime}=0\right)$. The latter amplitude is evaluated from Eq. (4) by putting $z=0$. Finally, the outgoing, diffracted field $E_{\ell}$ is calculated from the completely defined r.h.s. of Eq. (4).

The Bragg angle $\Theta_{\mathrm{B}}$ corresponds to the effective SAWinduced diffraction of MC polaritons with $p_{\|}= \pm k / 2$ and is given by $\sin \Theta_{\mathrm{B}}=\left[k /\left(2 k_{\text {opt }}\right)\right]\left\{1 \pm\left[\left(v_{\mathrm{s}} k\right) /\left(8 \varepsilon_{b} \omega_{\mathrm{T}}\right)\right] \times\right.$ $\left.\left[(c k) / \Omega_{\mathrm{x}}^{\mathrm{MC}}\right]^{2}\right\} \simeq k /\left(2 k_{\text {opt }}\right)$, where $k_{\text {opt }}=\omega / c$ is the wave vector of the external optical wave. In Fig. 2(a) we plot the reflection coefficient $\left|r\left(\omega, I_{\mathrm{ac}}\right)\right|^{2}$ of the incoming light incident at angle $\alpha=\Theta_{\mathrm{B}}$ on the SAW-driven MC
$\left(\nu_{\mathrm{SAW}}=1 \mathrm{GHz}\right)$. In this case the boundary at $p_{\|}=k / 2$ of the acoustically induced first Brillouin zone is probed (see Fig. 1). In the vicinity of $\omega=\omega_{\mathrm{LB}}^{\mathrm{MC}}(k / 2)$, with increasing $I_{\mathrm{ac}}$ the reflectivity changes its single-line shape for $I_{\mathrm{ac}}=0$ [the initial reflection spectrum is shown in Fig. 2(a) by the bold solid line] to a high contrast double-line shape with the separation $\Delta_{\mathrm{ac}}^{\mathrm{MC}}$ between two dips. Furthermore, a similar double-line structure, which refers to the threephonon-assisted transition, appears and develops with increasing $I_{\mathrm{ac}}$ for the reflectivity at $\omega \simeq \omega_{\mathrm{LB}}^{\mathrm{MC}}(3 k / 2)$ [see the inset of Fig. 2(a)]. Because the wave vector band $-k / 2 \leq$ $p_{\|} \leq k / 2$ can also be interpreted in terms of the SAWinduced reduced Brillouin zone, the incident optical wave probes the odd-order SAW-induced energy gaps (the areas marked in Fig. 1 by large solid and dashed circles refer to the $n=1$ and 3 transitions). The corresponding frequencydown-shifted -1 and -3 Bragg replicas give rise to the outgoing optical signal and are plotted in Fig. 2(b). The
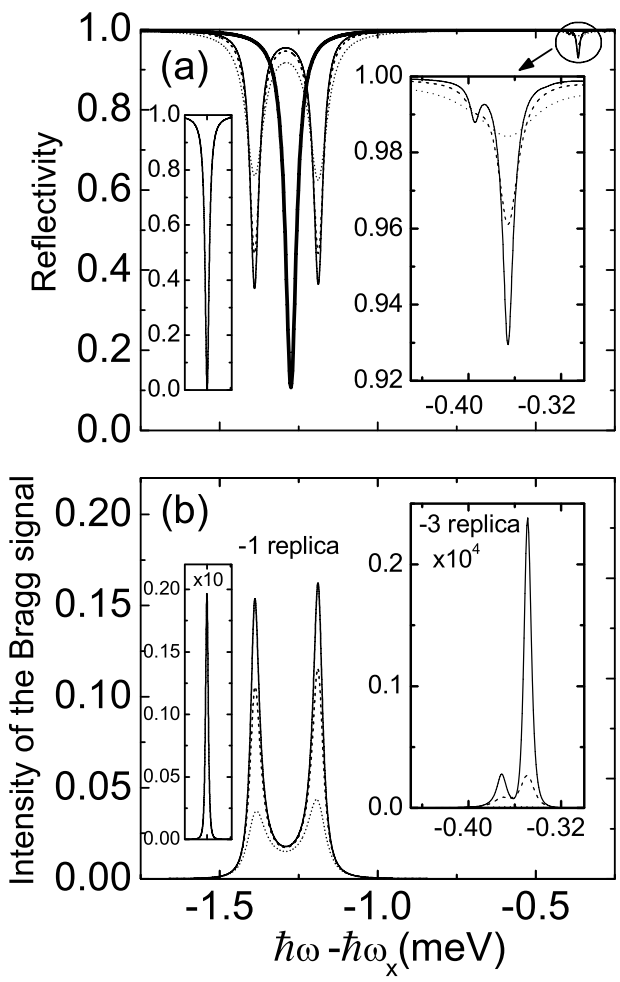

FIG. 2. The Bragg spectra of SAW-driven MC polaritons. The incident optical wave excites the MC polariton states at the r.h.s. boundary of the acoustically induced first Brillouin zone (see Fig. 1), i.e., $p_{\|}=k / 2$ and $\alpha=\alpha_{\mathrm{B}} \simeq 8.2^{\circ}$. (a) The reflection coefficient $\left|r\left(\omega, I_{\mathrm{ac}}\right)\right|^{2}$ of the optical wave against the frequency $\omega$. The thick solid line shows the initial polariton-mediated MC reflectivity for $I_{\mathrm{ac}}=0$ and $\hbar \gamma_{\mathrm{x}}=10 \mu \mathrm{eV}$. The thin lines refer to $\left|r\left(I_{\mathrm{ac}}=10 \mathrm{~W} / \mathrm{cm}^{2}\right)\right|^{2}$ for spectral vicinity of $n=1$ SAWphonon transition. Inset: $|r|^{2}$ for spectral vicinity of $n=3$ SAWphonon transition. (b) The -1 and -3 Bragg diffraction replicas normalized to $I_{\text {opt }}$. The 1.h.s. inset: -1 Bragg replica calculated by using the nonresonant $\chi_{\gamma}^{(2)}$ ac. The solid, dashed and dotted lines refer to the excitonic damping $\hbar \gamma_{\mathrm{x}}=\hbar / T_{2}=5,10$, and $30 \mu \mathrm{eV}$, respectively. 

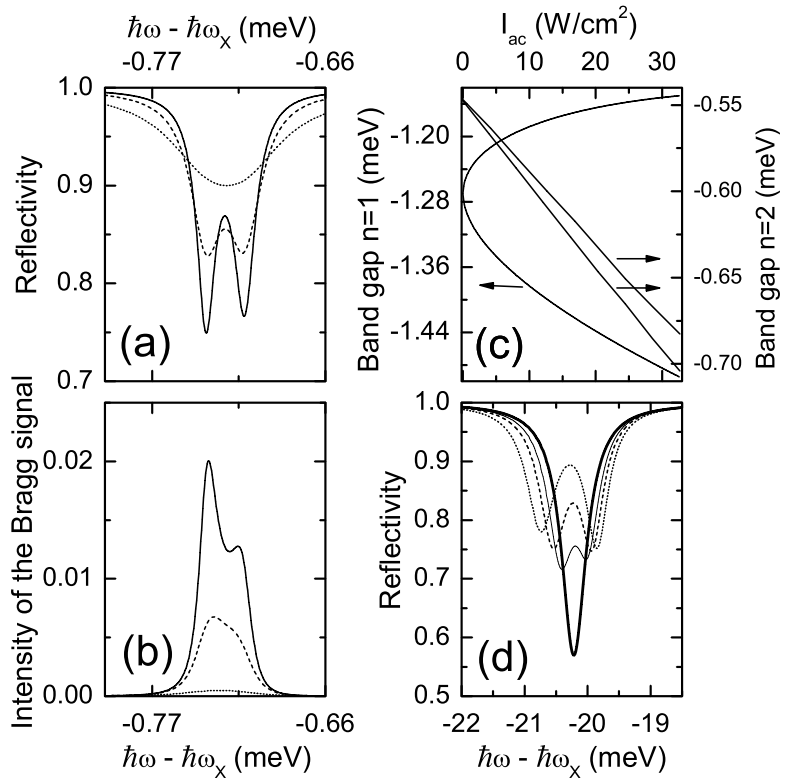

FIG. 3. The Bragg spectra of SAW-driven MC polaritons for $n=2$ transition: (a) The reflection coefficient $\left|r\left(\omega, I_{\mathrm{ac}}\right)\right|^{2}$ for $\alpha=16.8^{\circ}\left(p_{\|} \simeq k\right.$ and $\omega \simeq \omega_{\mathrm{LB}}^{\mathrm{MC}}(k)$, see Fig. 1$), \nu_{\mathrm{SAW}}=1 \mathrm{GHz}$ and $I_{\mathrm{ac}}=40 \mathrm{~W} / \mathrm{cm}^{2}$; (b) The frequency-down-converted -2 Bragg replica. The solid, dashed, and dotted lines refer to the excitonic damping $\hbar \gamma_{\mathrm{x}}=\hbar / T_{2}=5,10$, and $30 \mu \mathrm{eV}$, respectively; (c) The SAW-induced spike separation, i.e., the band gaps $\Delta_{\mathrm{ac}}^{\mathrm{MC}(\mathrm{n}=1)}$ and $\Delta_{\mathrm{ac}}^{\mathrm{MC}(\mathrm{n}=2)}$, against $I_{\mathrm{ac}}$. The Bragg spectroscopy at room temperature: (d) The specular reflectivity $|r|^{2}=$ $\left|r\left(\omega, I_{\mathrm{ac}}\right)\right|^{2}$ for $\alpha=\alpha_{\mathrm{B}}, \hbar \gamma_{\mathrm{x}}=1 \mathrm{meV}, \hbar \Omega_{\mathrm{x}}^{\mathrm{MC}}=20 \mathrm{meV}$ and MC detuning $\delta^{\mathrm{MC}}=-17 \mathrm{meV} . \nu_{\mathrm{SAW}}=1 \mathrm{GHz}$ and $I_{\mathrm{ac}}=0$ (thick solid line), $1 \mathrm{~kW} / \mathrm{cm}^{2}$ (thin line), $2 \mathrm{~kW} / \mathrm{cm}^{2}$ (dashed line), and $4 \mathrm{~kW} / \mathrm{cm}^{2}$ (dotted line).

camelback shape of the replicas follow the band gaps $\Delta_{\mathrm{ac}}^{\mathrm{MC}}$ and $\Delta_{\mathrm{ac}}^{\mathrm{MC}(\mathrm{n}=3)}$, respectively. In Fig. 3(a), $\left|r\left(\omega, I_{\mathrm{ac}}\right)\right|^{2}$ is shown for $\alpha \simeq 2 \Theta_{\mathrm{B}}$, so that the boundary at $p_{\|}=k$ of the second (extended) Brillouin zone is probed. The corresponding -2 Bragg replica is plotted in Fig. 3(b). In Fig. 3(c) we show the energy separation between two spikes in the -1 and -2 replicas. The separation is equal to $\Delta_{\mathrm{ac}}^{\mathrm{MC}} \propto I_{\mathrm{ac}}^{1 / 2}$ and $\Delta_{\mathrm{ac}}^{\mathrm{MC}(\mathrm{n}=2)} \propto I_{\mathrm{ac}}$, respectively.

In Figs. 2 and 3(a)-3(c) the Bragg spectroscopy of $\mathrm{SAW}$-driven MC polaritons is illustrated for the relatively small $\hbar \gamma_{\mathrm{x}} \lesssim 30 \mu \mathrm{eV}$ relevant to the helium temperatures $T \lesssim 10 \mathrm{~K}$. As shown in Fig. 3(d), the room temperature resonant acousto-optic effect can be realized in GaAs microcavities with giant Rabi splitting $\hbar \Omega_{\mathrm{x}}^{\mathrm{MC}} \simeq 20 \mathrm{meV}$ [15] and large negative MC detuning $\delta^{\mathrm{MC}} \lesssim-10 \mathrm{meV}$. The latter is necessary to suppress effectively the QWexciton damping $\hbar \gamma_{\mathrm{x}} \sim 1 \mathrm{meV}$, by the motional narrowing effect [in this case the exciton component is rather small, $\left.\varphi^{\mathrm{MC}}(k / 2) \lesssim 0.1\right]$.

Bragg diffraction, due to the nonresonant $\chi_{\gamma-\text { ac }}^{(2)}$, has been observed for an optical wave guided by SAW-driven semiconductor layers [16-18]. The one-phonon diffraction replica - 1, calculated for the GaAs-based MC with the use of $\chi_{\gamma-\mathrm{ac}}^{(2)}$, is plotted in the 1.h.s. inset of Fig. 2(b). In this case the acoustically induced main stop gap in the MCphoton spectrum is very small and completely screened by the radiative damping, so that it cannot be seen in the Bragg signal as a camelback structure. Note that the SAW intensities $I_{\mathrm{ac}}$ we discuss are much less than those used to acoustically ionize, by the piezoelectric effect, the excitons in GaAs structures [19,20].

We appreciate valuable discussions with Yu. Kosevich, P. Littlewood, and W. Sohler. Support of this work by Grant-in-Aid No. 15540311 of the Ministry of Education of Japan, Toyota Phys. \& Chem. Institute, and EU RTN Project No. HPRN-2002-00298 is gratefully acknowledged.

*Present address: Fukui University of Technology, 9108505 Japan.

[1] L. Brillouin, Actual. Sci. Ind. 59, 1 (1933).

[2] G. Blanch, in Handbook of Mathematical Functions, edited by M. Abramowitz and I. A. Stegun (Dover Publications, New York, 1965), p. 721.

[3] J. Wilson and J. F. B. Hawkes, Optoelectronics (Prentice Hall International, New Jersey, 1983), Section 3.8.

[4] A. Korpel, Acousto-Optics (M. Dekker, Inc., New York, 1997), second ed., Chap. 2 and 3.

[5] L. Brillouin, Ann. Phys. (Paris) 17, 88 (1922).

[6] R. S. Chu and T. Tamir, J. Opt. Soc. Am. 66, 220 (1976); R. S. Chu and J. A. Kong, J. Opt. Soc. Am. 70, 1 (1980).

[7] A. L. Ivanov and P. B. Littlewood, Phys. Rev. Lett. 87, 136403 (2001). Because of a misprint, the factor 2 in the last term on the left-hand side of Eq. $4 \mathrm{~b}$ should be replaced by 4 .

[8] A. L. Ivanov and P. B. Littlewood, Semicond. Sci. Technol. 18, S428 (2003). In this paper only the piesoelectric exciton-phonon interaction is included for the SAWinduced scattering.

[9] M.S. Skolnick, T.A. Fisher, and D.M. Whittaker, Semicond. Sci. Technol. 13, 645 (1998).

[10] J. J. Baumberg, Phys. World 15, 37 (2002).

[11] A. Kavokin and G. Malpuech, Cavity Polaritons (Elsevier, Amsterdam, 2003).

[12] C. Campbell, Surface Acoustic Wave Devices and Their Signal Processing Applications (Academic, New York, 1989), Chap. 2.

[13] S. H. Simon, Phys. Rev. B 54, 13878 (1996).

[14] K. Cho, Optical Response of Nanostructures: Microscopic Nonlocal Theory (Springer Verlag, Heidelberg, 2003), Section 3.4; J. Lumin. 102/103, 232 (2003).

[15] J. Bloch et al., Appl. Phys. Lett. 73, 1694 (1998).

[16] L. Kuhn et al., Appl. Phys. Lett. 17, 265 (1970).

[17] K. W. Loh et al., Appl. Opt. 15, 156 (1976).

[18] D. Ciplys et al., Appl. Phys. Lett. 80, 1701 (2002).

[19] C. Rocke et al., Phys. Rev. B 57, R6850 (1998); Phys. Rev. Lett. 78, 4099 (1997).

[20] P. V. Santos, M. Ramsteiner, and F. Jungnickel, Appl. Phys. Lett. 72, 2099 (1998). 